\title{
Recasting Radiographic Image Data for Accurate Abel Inversion
}

\author{
S. E. Mitchell, M. Howard, and A. Luttman \\ National Security Technologies, LLC \\ Las Vegas, NV 89193
}

\section{Abstract}

One the primary goal of quantitative X-ray radiography is to compute the densities of objects in the scene being imaged. The most common approach to doing this is to use radiation transport modeling to simulate a forward radiograph that best matches the measured image. When the object being imaged is cylindrically symmetric, it is possible to instead infer object densities directly from the measured data, viewing the image as a single tomographic projection of the object. This is a two-stage process, as the intensity data must be converted to areal density, then the areal density is mapped to volumetric density by the inverse Abel transform. Abel inversion is an ill-posed problem, which means that it is sensitive both to noise in the measured intensity image and to errors in the conversion from intensity to areal density. In this work we develop a novel approach to computing areal density from intensity and show how the improved areal density calculations result in significantly improved volumetric density estimates. We further show correlation between the recasting model parameters and presumed source spectrum shape and compare results with experimental observations.

\section{Keywords-Radiography, Abel, Density} Reconstruction

\section{INTRODUCTION}

Radiation generating devices (RGD) produce X-rays which are commonly used to interrogate an object to infer absolute quantitative information, such as an object's density, from radiographic image obtained of the scene. The U.S. Department of Energy research complex has several highenergy X-ray sources used for quantitative radiography, such as RITS-6 [1,2] at Sandia National Laboratories, DARHT at Los Alamos National Laboratory [3,4], and Cygnus at the Nevada National Security Site $[5,6]$. For each of these distinct RGD systems, X-rays produced penetrate through an object of interest under investigation, and those X-rays not absorbed by objects of interest deposit any remaining energy into a scintillator, which fluoresces in the visible spectrum in approximately linear response to the X-rays incident upon it. The visible light emitted by the scintillator is typically captured by an optical imaging system such as a chargecoupled device (CCD) camera. Figure 1 shows a basic schematic of the imaging process for a cylindrically symmetric object consisting of three concentric layers, each composed of different materials with differing densities. In this configuration, the values at each pixel in the image as captured from the CCD array is directly proportional to X-ray transmission through the object in the radiographic field of view (FOV). Intensity, as observed at the CCD array plane and consequently mapped back to an emitting point on the scintillator is then approximately the line integral of the X-ray attenuation along the line of site from the X-ray source. If the object is radially, or in this case cylindrical symmetric about a central axis, then computing its density profile is the singleprojection tomographic reconstruction problem known as Abel inversion.

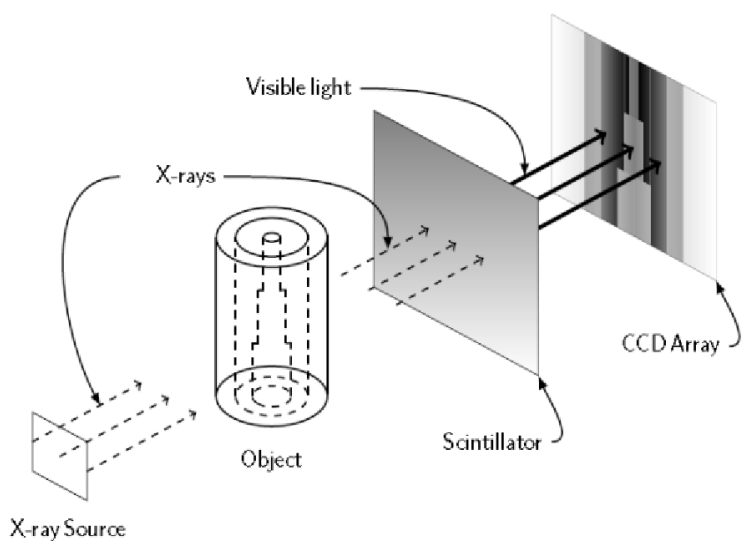

Figure. 1 Abel inversion is a method for reconstructing the density profile of an object with cylindrical (or spherical) symmetry, such as the one seen in the figure above, from a single tomographic projection.

\section{A. Converting Intensity to areal density}

Images captured by X-ray imaging systems like those described above are direct measures of the intensity of visible light emitted by the scintillator [7], which, as also noted, is an indirect measure of photon attenuation through matter within the radiographic FOV. The materials' photon attenuation properties comprise of the mass attenuation coefficient, specific to the object's material, mass density of the object, and the thickness or the ray trace length through the object. The measured intensity, I, can be converted to areal density, $\sigma$, using a measured transmission curve [8], but it is common in practice to use the approximation

$$
\sigma \propto-\log _{n}\left(\frac{I}{I_{\max }}\right),
$$

where $n$ is the log base and the scale of proportionality is constant across the spatial domain $[9,10]$. This is equivalent 
to assuming that photons are attenuated exponentially, a result that obeys Beer's Law [11], which is an accurate model for monoenergetic X-rays and materials with similar X-ray crosssections. However, many pulsed power X-ray systems typically produce a non-monoenergetic Bremsstrahlung spectrum [12], and that the conversion from intensity to areal density, rather than the actual Abel inversion, is the primary source of error in density reconstructions from real data.

\section{RECASTING BASIS MODEL}

First, we address the primary source of error in density reconstructions by focusing our attention on a generic Bremsstrahlung source and its impact with respect to a radiographic image. Unlike a monochromatic X-ray source obeying a simple Beer's law attenuation relation, the transmission is proportional to a Bremsstrahlung source's effective attenuated contribution and may be represented as a sum of weighted exponentials

$x m t \propto w_{1} B_{1} e^{-\mu_{1} \sigma}+w_{2} B_{2} e^{-\mu_{2} \sigma}+\ldots+w_{N} B_{N} e^{-\mu_{N} \sigma}$,

(2)

where $x m t$ is the observed transmission through an object, $w_{\mathrm{i}}$ are the individual weights of the spectrum at energy i, $\mu_{i}$ are the mass attenuation coefficients [13] at energy i, $B_{i}$ is the buildup factor, and $\sigma$ is the areal density. Energy $N$ represents the limiting case or end-point energy. In a similar formulism, $x m t$ as determined through a transmission curve may be represented in a continuous sense, complimentary to the discrete case in Eq. 2, as

$$
x m t \propto B(\sigma) e^{-\mu_{e f f} \sigma},
$$

where $\mu_{\text {eff }}$ is some weighted average of all mass attenuation coefficients spanning the working energy range and represented as a single effective mass attenuation coefficient and $B(\sigma)$ is an effective buildup term over the areal density range of interest.

As an example, transmission curve data from a recent Cygnus radiographic shot suggest that Eq. 3 formulism takes a form that can be well fit by a Gamma function. In particular, $B(\sigma)$ is proportional to the form $\sigma^{-c}{ }_{2}$ [14] in some limited range of $\sigma$ and $c_{2}$ is a real number fitted to the transmission curve data and $\mu_{e f f}$ becomes another fitting parameter $c_{l}$. Thus, for Cygnus, $x m t$ as depicted in Eq. 3 can be modeled as

$$
x m t \propto \sigma^{c_{2}} e^{-c_{1} \sigma} \text {. }
$$

It was reasoned from Eq. 1 that $\sigma$ is proportional to $-\log _{n}(I)$. Moreover, we can claim $\sigma=-c_{3} \log _{n}(I)$, where $c_{3}$ is a scaling factor consistent across the spatial domain of the image and I' is already normalized by $I_{\max }$. So, simple substitution into Eq. 4 yields

$$
\begin{aligned}
x m t \propto\left(-c_{3} \log _{n}\left(I^{\prime}\right)\right)^{c_{2}} e^{-c_{1}\left(-c_{3} \log _{n}\left(I^{\prime}\right)\right)}, \\
x m t=\left(-c_{3} \log _{n}\left(I^{\prime}\right)\right)^{c_{2}} I^{\prime c_{1} c_{3}},
\end{aligned}
$$

finally, $x m t$ is scaled by $c_{4}$ as

$$
x m t=c_{4}\left(-c_{3} \log _{n}\left(I^{\prime}\right)\right)^{c_{2}} I^{c_{1} c_{3}},
$$

In this section, we first present a test case by introducing a true one dimensional forward Abel projection of an ideal density distribution (also one dimensional) from a conceptual embedded cylindrical embedded object. Next, we compare a one-dimensional forward and inverse Abel operation, along with the recast model prescription in this report, on a synthetic ray trace section on the same conceptual embedded cylindrical embedded object. Finally, we compare a one dimension forward and inverse Abel operation on a section of an actual Cygnus radiograph, consistent with the conceptual configuration and under the similar operations and recast prescription previously described, and compare results to truth.

\section{TRUTH: FORWARD ABEL OF AN IDEAL DENSITY DISTRIBUTION}

Starting with a conceptual embedded cylindrical embedded object as depicted in Fig. 2, we may begin directly with an assumed density profile in pixel space with respect to the CCD array plane shown earlier in Fig. 1. Such a density profile is identified in Fig. 3 as the blue plot with units described on the left side ordinate.
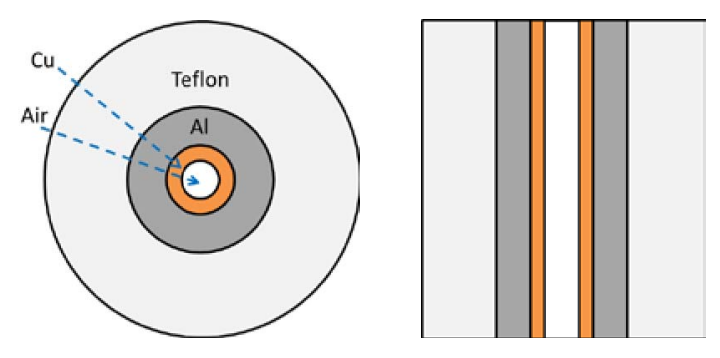

Figure. 2 [left] Cartoon illustration of cylindrical object in $\rho-\theta$ plane with material identification. [Right] $2-\mathrm{D}$ projected slice through the $z-\rho$ plane of the same object

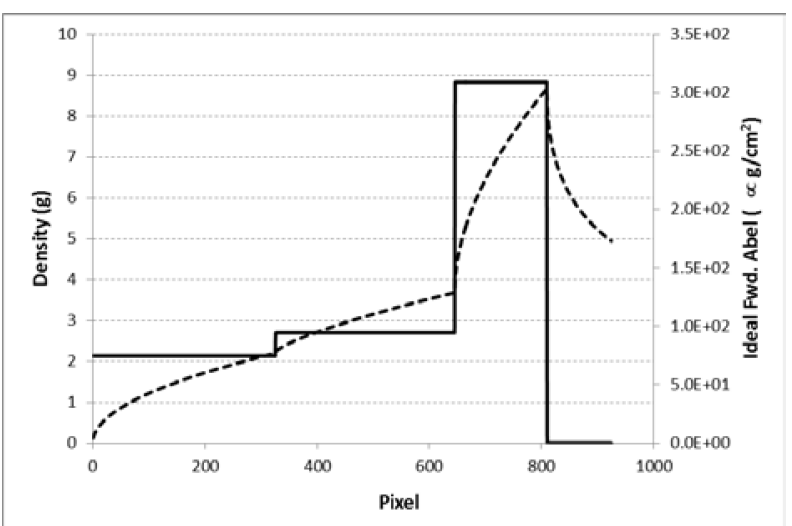

Figure. 3 [BLACK] Ideal density profile plot in pixel coordinates with respect to the $\mathrm{CCD}$ array plane. [DASH] Ideal forward Abel of a 1-D line-out from the center line of the object as observed wrt CCD array plane.

\section{III.RESULTS}

Identify applicable sponsor/s here. If no sponsors, delete this text box (sponsors). 
The forward Abel operation [15] is shown here for reference

$$
\begin{aligned}
& x m t(x)=K * \rho(x), \\
& x m t(x) \propto e^{-\mu \sigma}
\end{aligned}
$$

with its inverse as

$$
\rho(x)=-\frac{1}{\pi} K * \frac{d}{d x} x m t(x)
$$

where $K$ is the Abel convolution kernel, $\operatorname{xmt}(x)$ is the transmission observed wrt to the CCD array plane, and $\rho(x)$ is the 1-D spatial dependent density distribution.

\section{V.SYNTHETIC: INVERSE ABEL OF RAY TRACE MODEL}

Fig. 4 below shows the qualitative comparison of all three forward Abel representations; (1) the forward Abel of an ideal 1-D line-out from the center line of the object as observed wrt CCD array plane, (2) scaled ray-trace plot, and (3) scaled and recasted ray-trace plot.

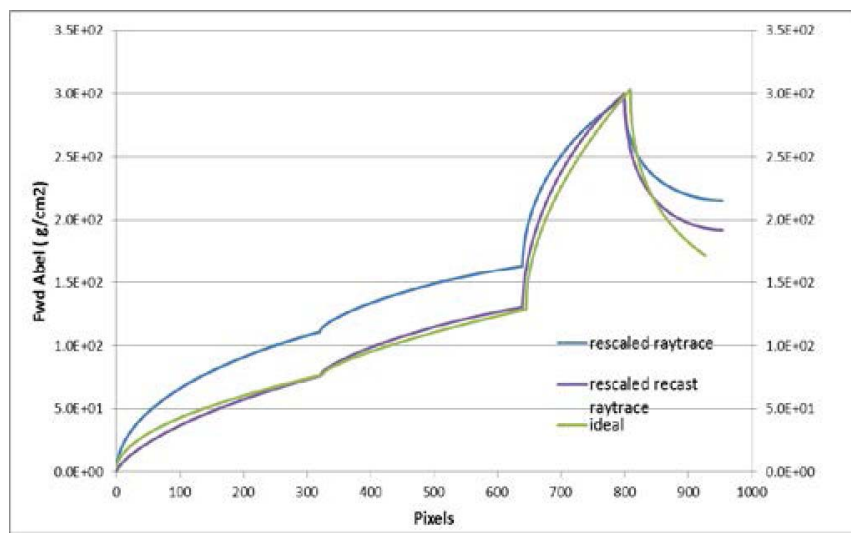

Figure. 4 [GREEN] Ideal forward Abel of a 1-D line-out from the center line of the object as observed wrt CCD array plane [BLUE] Scaled ray-trace plot [PURPLE] Scaled and recasted ray-trace plot.

Fig. 5 below shows the qualitative comparison of all three inverse Abel density representations; (1) an ideal inverse Abel of a 1-D line-out from the center line of the object as observed wrt CCD array plane, (2) inverse Abel of the scaled ray-trace plot, and (3) inverse Abel of the scaled and recasted ray-trace plot.

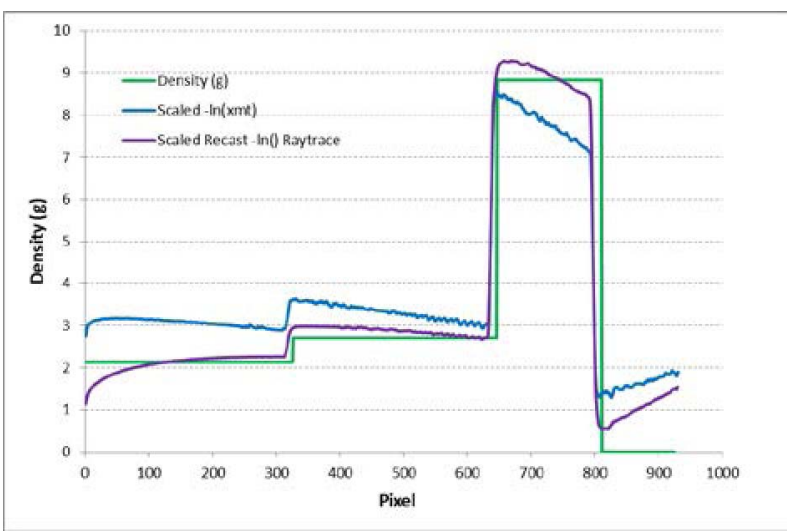

Figure. 5 [GREEN] Ideal inverse Abel of a 1-D line-out from the center line of the object as observed wrt CCD array plane [BLUE] inverse Abel of the scaled ray-trace plot [PURPLE] Inverse Abel of the scaled and recasted ray-trace plot.

There is much better overall agreement between the inverse Abel scaled and recasted ray-trace (purple plot in Fig 5) data and the ideal density profile as opposed to the un-recasted data (blue plot in fig 5). The inverse Abel of the scaled ray-trace data largely underestimates higher densities while overestimates lower densities is often the disagreement trait encountered in many density reconstruction schemes. A table summarizing the mean difference and fractional error agreement from truth per density layer step is outlined at the end of the result section

The recasting fitting parameters, $c_{2}$ and $c_{l}$ in particular, reveal characteristic information regarding the source and ray-trace information. $c_{2}$ and $c_{1}$ are zero and non-zero respectively indicating correctly that synthetic ray-trace data is monochromatic and obeys a single Beer law relation. A table summarizing the coefficient values for $c_{1}, c_{2}$, and $c_{3}$ is also outlined at the end of the result section

\section{SYNTHETIC: INVERSE ABEL OF MCNP MODEL}

An MCNP[16][17] calculation was performed to simulate the radiation transport of a $5 \mathrm{Mev}$ end-point energetic Kramer[18] spectrum through the nested cylinders in lieu of an actual RGD producing the desired data. Fig. 6 below shows the qualitative comparison of all three forward Abel representations; (1) the forward Abel of an ideal 1-D line-out from the center line of the object as observed wrt CCD array plane, (2) inverse Abel of the scaled simulated data plot, and (3) inverse Abel of the scaled and recasted simulated data plot. 


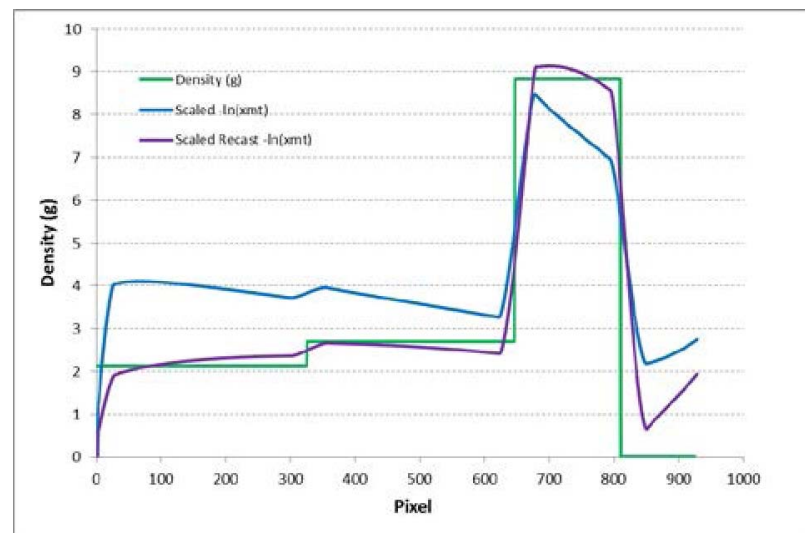

Figure. 6 [GREEN] Ideal inverse Abel of a 1-D line-out from the center line of the object as observed wrt CCD array plane [BLUE] inverse Abel of the scaled MCNP simulated plot [PURPLE] Inverse Abel of the scaled and recasted MCNP simulated plot.

There is general good overall agreement between the inverse Abel scaled and recasted MCNP simulated data (purple plot in Fig 6) and the ideal density profile as opposed to the unrecasted data (blue plot in fig 6). Again, the inverse Abel of the scaled ray-trace data largely underestimates higher densities while overestimates lower densities is often the disagreement trait encountered in many density reconstruction schemes.

Again, the recasting fitting parameters, $c_{2}$ and $c_{1}$ in particular, reveal characteristic information regarding the source and ray-trace information. $c_{2}$ and $c_{1}$ are both non-zero respectively indicating correctly, according to the model premise, that the simulated image data produced with a Kramer spectrum is not monochromatic and largely obeys a relation as laid out earlier in section II.A.

\section{REAL DATA: INVERSE ABEL OF CYGNUS IMAGE DATA}

Fig. 7 below shows an actual radiograph image of the real embedded cylindrical object defined in our test case earlier.

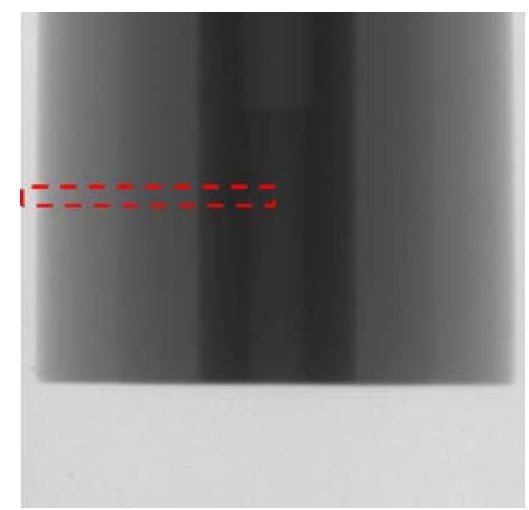

Figure. 7 Actual radiograph image of the real embedded cylindrical object defined in our test case earlier. The dotted red box overlay indicates approximate region where a line-out (column collapse) was performed to extract 1-D data.

The red dotted box indicates the approximate region where a line-out operation was performed to extract 1-D data for other subsequent operations.

Fig. 8 below shows the qualitative comparison of all three forward Abel representations; (1) the forward Abel of a 1-D line-out from the center line of the object as observed wrt CCD array plane, (2) scaled real data plot, and (3) scaled and recasted real data plot.

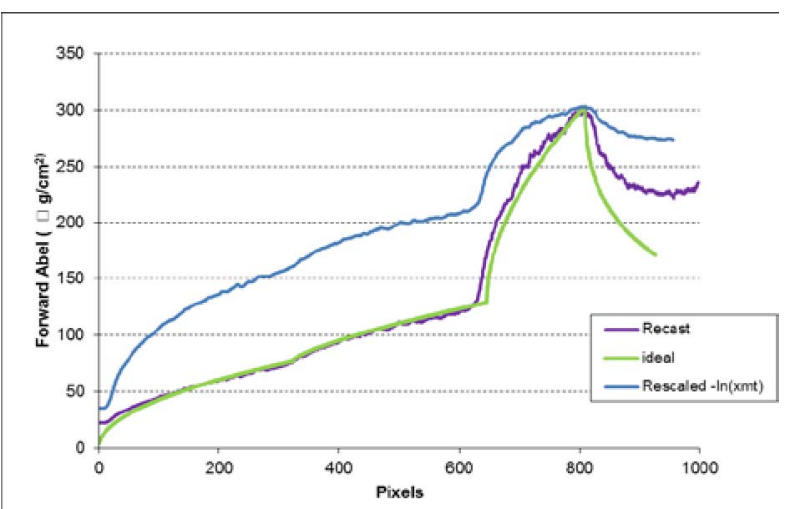

Figure. 8 [GREEN] Ideal forward Abel of a 1-D line-out from the center line of the object as observed wrt CCD array plane [BLUE] Scaled real data plot [PURPLE] Scaled and recasted real data plot.

Fig. 9 below shows the qualitative comparison of all three inverse Abel density representations; (1) an ideal inverse Abel of a 1-D line-out from the center line of the object as observed wrt CCD array plane, (2) inverse Abel of the scaled real data plot, and (3) inverse Abel of the scaled and recasted real data plot.

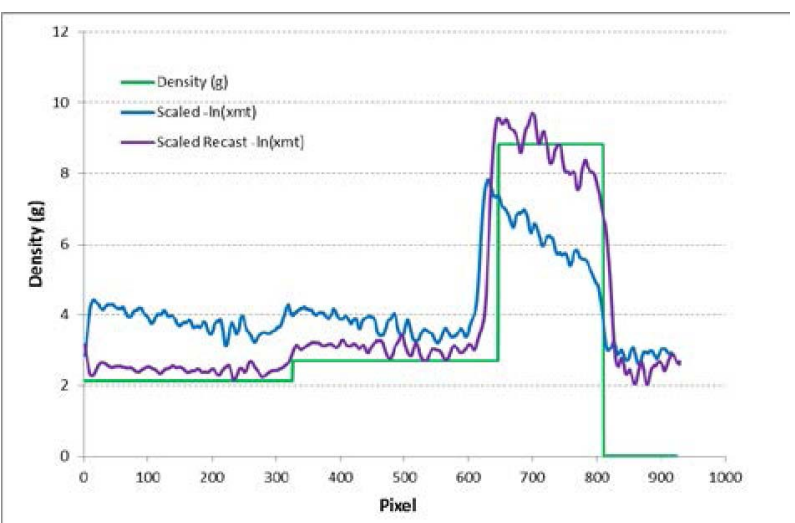

Figure. 9 [GREEN] Ideal inverse Abel of a 1-D line-out from the center line of the object as observed wrt CCD array plane [BLUE] inverse Abel of the real data plot [PURPLE] Inverse Abel of the scaled and recasted real data plot. 
There is general good overall agreement between the inverse Abel scaled and recasted real data (purple plot in Fig 9) and the ideal density profile as opposed to the un-recasted data (blue plot in fig 9). Again, the inverse Abel of the scaled raytrace data largely underestimates higher densities while overestimates lower densities is often the disagreement trait encountered in many density reconstruction schemes.

Again, the recasting fitting parameters, $c_{2}$ and $c_{1}$ in particular, reveal characteristic information regarding the source and raytrace information. $\mathrm{c}_{2}$ and $\mathrm{c}_{1}$ are both non-zero respectively indicating correctly, according to the model premise, that the real image data produced at the Cygnus facility is not monochromatic, hence Bremsstrahlung, and largely obeys a relation as laid out earlier in section II.A.

\section{RESULT SUMMARY}

Table 1 below summarizes both the coefficients to the recasting model and comparative errors for the representative cases outlined earlier in this paper.

\begin{tabular}{|c|c|}
\hline \multicolumn{2}{|l|}{ Ray Trace } \\
\hline Recast parameters $c_{1}, c_{2}, c_{3}, c_{4}$ & $-0.28,0.6,1.4,37.5$ \\
\hline $\begin{array}{l}\text { Error } 1^{\text {st }}, 2^{\text {nd }}, 3^{\text {rd }}, \text { and } 4^{\text {th }} \text { density } \\
\text { layer (NO RECAST) }\end{array}$ & $\begin{array}{llll}0.45, & 0.23, & -0.11, & \text { and } \\
1,240 & & & \end{array}$ \\
\hline $\begin{array}{l}\text { Error } 1^{\text {st }}, 2^{\text {nd }}, 3^{\text {rd }} \text {, and } 4^{\text {th }} \text { density } \\
\text { layer (RECAST) }\end{array}$ & $\begin{array}{l}0.022,0.074,0.018 \text {, and } \\
714\end{array}$ \\
\hline \multicolumn{2}{|l|}{ MCNP Simulation } \\
\hline Recast parameters $c_{1}, c_{2}, c_{3}, c_{4}$ & $-1.15,0.9,0.7,122$ \\
\hline $\begin{array}{l}\text { Error } 1^{\text {st }}, 2^{\text {nd }}, 3^{\text {rd }}, \text { and } 4^{\text {th }} \text { density } \\
\text { layer (NO RECAST) }\end{array}$ & $\begin{array}{llll}0.88, & 0.35, & -0.11, & \text { and } \\
1,790 & & & \end{array}$ \\
\hline $\begin{array}{l}\text { Error } 1^{\text {st }}, 2^{\text {nd }}, 3^{\text {rd }} \text {, and } 4^{\text {th }} \text { density } \\
\text { layer (RECAST) }\end{array}$ & $\begin{array}{l}0.065,-0.039,0.024 \text {, and } \\
625\end{array}$ \\
\hline \multicolumn{2}{|l|}{ Real Image Data } \\
\hline Recast parameters $c_{1}, c_{2}, c_{3}, c_{4}$ & $-1.5,0.01,1.1,16$ \\
\hline $\begin{array}{l}\text { Error } 1^{\text {st }}, 2^{\text {nd }}, 3^{\text {rd }}, \text { and } 4^{\text {th }} \text { density } \\
\text { layer (NO RECAST) }\end{array}$ & $\begin{array}{llll}0.77, & 0.38, & -0.31, & \text { and } \\
2,290 & & & \\
\end{array}$ \\
\hline $\begin{array}{l}\text { Error } 1^{\text {st }}, 2^{\text {nd }}, 3^{\text {rd }} \text {, and } 4^{\text {th }} \text { density } \\
\text { layer (RECAST) }\end{array}$ & $\begin{array}{l}0.14,0.089,0.00068 \text {, and } \\
1,820\end{array}$ \\
\hline
\end{tabular}

Table 1. Result summary.

\section{CONCLUSIONS}

We have shown considerable error reduction in density reconstruction by prescribed method of recasting the image data in one nested cylindrical symmetric configuration. In majority cases, the improvement is of order magnitude. The recasting parameters for each of the test cases suggest high correlation to source profile (e.g. monoenergetic, synthetic distribution, or Bremsstrahlung) and implicitly, the RGD and transport of the source term.

Next, we plan on studying the stability of the recasting image data model by exploring the mean and variance for each of the recasting parameters for a variety of nested cylindrical and spherical symmetric configurations and source terms.

\section{ACKNOWLEDGEMENTS}

We would like to thank our DESS management, Joshua Friedman, Michael Kelly, Darryl Droemer, Jim Gatling, and Steve Goldstein, for their continuous support of our work and efforts. We also would like to thank our LANL colleagues and Cygnus community, Todd Haines and John Smith in particular, for their support.

This manuscript has been authored by National Security Technologies, LLC, under Contract No. DE-AC5206NA25946 with the U.S. Department of Energy and supported by the Site-Directed Research and Development Program. The United States Government retains and the publisher, by accepting the article for publication, acknowledges that the United States Government retains a non-exclusive, paid-up, irrevocable, world-wide license to publish or reproduce the published form of this manuscript, or allow others to do so, for United States Government purposes. The U.S. Department of Energy will provide public access to these results of federally sponsored research in accordance with the DOE Public Access Plan (http://energy.gov/downloads/doe-public-access-plan). DOE/NV/25946--2494

\section{REFERENCES}

[1] S. Portillo, B. V. Oliver, S. R. Cordova, N. Bruner, D. Ziska, and D. Rovang, "Demonstration of the selfmagnetic-pinch diode as an X-ray source for ash corepunch radiography", Tech. Report SAND2007-6324, Sandia National Laboratories, 2007.

[2] K. D. Hahn et al, "Overview of self megnetically pinched-diode investigations on RITS-6", IEEE Transactions on Plasma Science, 38 (2010), pp. 26522662.

[3] Kevin Roark, "LANL fires a DAHRT", DOE Pulse, (2009).

[4] R. D. Scarpetti et al, "Status of the DARHT second axis accelerator at the Los Alamos National Laboratory", Proceedings of the PAC07, 2007, pp. 831-835.

[5] J. Smith, R. Carlson, and et al., "Cygnus dual beam radiography source", Pulsed Power Conference, 2005 IEEE, 2005, pp. 334-337.

[6] J. Smith and et. al, "Cygnus performance in subcritical experiments", 16th IEEE International Pulsed Power Conference, 2007, pp. 685-688.

[7] S. Baker, et al, "Scintillator efficiency study with mev xrays", Proc. SPIE, 9213 (2014), pp. 92130H-92130H-10.

[8] A.N. Parmar, D.D.E. Martin, M. Bavdaz, F. Favata, E. Kuulkers, G. Vacanti, U. Lam mers, A. Peacock, and B.G. Taylor, "The low-energy concentrator spectrometer on-board the bepposax x-ray astronomy satellite", Astron. Astrophys. Suppl. Ser., 122 (1997), pp. 309-326

[9] Thomas J. Asaki, Patrick R. Campbell, Rick Chartrand, Collin E. Powell, Kevin R. Vixie, and Brendt E. Wohlberg, "Abel inversion using total-variation regularization", Applications, Inverse Problems in Science and Engineering, 14 (2006), pp. 873-885.

[10] T. J. Asaki, R. Chartrand, K. R. Vixie, and B. Wohlberg, "Abel inversion using total variation regularization", Inverse Problems, 21 (2005), pp. 1895-1903. 
[11] A. Beer, "Bestimmung der Absorption des rothen Lichts in farbigen Flüssigkeiten", Annalen der Physik und Chemie, vol. 86, pp. 78-88.

[12] E. Haug and W. Nakel, "The elementary process of bremsstrahlung", vol. 73 of Scientific LectureNotes in Physics, World Scientific, 2004.

[13] J. H. Hubbell, , "Bibliography of Photon Total Cross Section (Attenuation Coefficient) Measurements $10 \mathrm{eV}$ to 13.5 GeV", 1907-1993, NISTIR 5437.

[14] Y. Harima, Y. Sakamoto, S. Tanaka, and M. Kawai,"Validity of the Geometrical Progression Formula in Approximating Gamma-Ray Buildup Factors," Nucl. Scì. Eng. 94, 24-35 (1986).
[15] R. Bracewell, "The Fourier Transform and its Applications", McGraw-Hill Inc., Second Ed., 1978.

[16] J. F. Briesmeister, ed., "MCNPTM - A General Monte Carlo N-Particle Transport Code, Version 4C," Los Alamos National Laboratory report LA-13709-M (March 2000).

[17] G. W. McKinney, T. E. Booth, J. F. Briesmeister, L. J. Cox, R. A. Forster, J. S. Hendricks, R. D. Mosteller, R. E. Prael, and A. Sood, "MCNP Applications for the 21st Century,"Proceedings of the 4th International ConfTokyo, Japan, September 4-7 (2000).

[18] H. A. Kramers, 1923 Phil. Mag. 46 836-71 